\title{
What's at the Heart of Anorexia Nervosa? Reconsidering the Physiology of Bradycardia in Anorexia Nervosa
}

James A. J. Heathers, Ph.D. ${ }^{a}$, Jason Nagata, M.D., M.Sc ${ }^{\text {b }}$, Stuart B. Murray, Ph.D. ${ }^{\text {c }}$

a Bouvé College of Health Sciences, Northeastern University, Boston, MA

${ }^{\mathrm{b}}$ Department of Pediatrics, University of California, San Francisco, San Francisco, CA

${ }^{\mathrm{c}}$ Department of Psychiatry, University of California, San Francisco, San Francisco, CA

Correspondence: Dr Stuart B. Murray, Department of Psychiatry, University of California, San Francisco, 401 Parnassus Avenue, San Francisco, CA, 94143, United States.

Email: stuart.murray@ucsf.edu

Keywords: anorexia nervosa; bradycardia; cardiac function; eating disorders

Word Count: 1,793 


\begin{abstract}
Anorexia nervosa (AN) is among the most pernicious, and lethal, of psychiatric disorders, in which starvation-induced medical instability often requires immediate hospitalization. In mitigating this medical risk, current guidelines posit that bradycardia, defined as a resting heart rate of less than 50 beats per minute, may serve as a biomarker from which we can infer medical instability in those with AN, with those meeting this criterion requiring urgent hospitalization, medical stabilization and refeeding. However, alongside dietary starvation, a central feature of AN relates to cardiovascular exercise, which is often excessively undertaken as a means to further augment weight loss. Crucially, frequent and intense cardiovascular exercise may also lower one's resting heart rate, leaving two potential mechanisms underpinning bradycardia in those with AN, which potentially portend differential risk profiles. Here we briefly review the potential co-contribution of exercise and starvation to bradycardia in those with $\mathrm{AN}$, and suggest that the precise delineation of factors underpinning bradycardia in those with $\mathrm{AN}$, and the relative degree of risk therein, is crucial in the precision management of AN.
\end{abstract}




\section{What's at the Heart of Anorexia Nervosa? Reconsidering the Physiology of Bradycardia in Anorexia Nervosa}

Anorexia nervosa (AN) is among the most pernicious of psychiatric disorders. Cardiovascular medical complications are seen in up to $80 \%$ of cases [1,2], and sudden cardiac-related death ranks as the highest cause of death in AN, excluding suicide. In managing the acute medical risk presented by $\mathrm{AN}$, bradycardia (a resting heart rate of less than 50 beats per minute) is thought to be a key indicant of medical instability, and treatment guidelines support immediate admission [3]. Bradycardia is present in up to $70 \%$ of AN presentations [4].

Mechanistically, it is presumed that in those with AN, bradycardia is a physiological adaptation designed for self-preservation during a state of starvation, since an increase in cardiovagal tone decreases cardiac work by reducing output to conserve energy [5]. In keeping, hospital-based treatment of acute AN is typically oriented towards medical stabilization (indicated by cardiovascular stabilization) [6], via intensive nutritional refeeding to counter the effects of starvation.

However, inferring cardiac-related risk from bradycardia may be problematic, since starvation is not the sole possible source of bradycardia in AN. Here we explore the unexamined influence of athletic bradycardia, a generally benign condition which is a component of adaptive and exerciseinduced cardiac remodelling. In athletes, the volume and pressure loads of increased internal and external cardiac work over time cumulatively result in a series of characteristic modifications to the heart and circulatory system, which include increased chamber and ventricular size, right bundle branch block, junctional escape rhythm, etc. in addition to bradycardia [7]. Crucially, these potentially discrepant sources of bradycardia may confer different degrees of short-term medical risk.

\section{Exercise and Anorexia Nervosa: Dual Pathways into Bradycardia?}

In considering the potential overlap between athletic pursuits and AN psychopathology, it is evident that athletic bradycardia could overlap with AN symptomatology. Highlighting this potential overlap, up to $80 \%$ of those with AN demonstrate compulsive, obsessional, intense cardiovascular 
exercise in attempting to modify shape and weight [8]. However, exercise is a sparsely researched topic both in eating disorders in general and within AN specifically, despite a strong call to increase research interest [9], and recent work showing the convergent validity of the Compulsive Exercise Test with more traditional psychometric inventories [10]. Likewise, compulsive exercise shows a moderate relationship with eating disorder psychopathology, indicating the benefit of addressing exercise as a treatment modality [11]. The nascent nature of this research area means specific and precise related mechanisms such as the subcomponents of bradycardia in AN have never been previously explicated.

In doing so, several lines of evidence warrant consideration:

1. Exercise profiles in $\mathbf{A N}$ are typically appropriate. Athletes performing endurance-based exercise with high dynamic demands (i.e. running, cycling) show markedly slower baseline heart rates than matched controls, while strength athletes do not [12]. Exercise profiles in AN are intricately intertwined with the core symptom profile, as exercise is often co-opted in the drive for thinness (although exercise does not necessarily present as a compensatory behavior), and performed to expend calories and thus augment the pursuit of weight loss. The most common exercise patterns in AN are similarly rooted in endurance-based exercise (running, high-repetition calisthenics, walking, soccer, swimming) [13], which may support the development of reduced resting heart rate.

2. The exercise volume and intensity in AN meets the necessary criteria for the development of athletic bradycardia. Athletic-related heart changes are typically observed in a cohort performing more than 3 hours exercise per week [12], but are more commonly observed at a training load of 4-8 hours per week (hrs. pw) of intense exercise [7]. Exercise habits in AN feature similar training loads (males $=8.3 \mathrm{hrs}$. pw; females $=6.82 \mathrm{hrs}$. pw) and frequency (males $=5.58$ bouts pw; females $=5.42$ bouts pw), although these figures may be under-reported to decrease perceived ED severity, and to increase perceived compliance with instructions not to exercise [13].

3. The total exercise duration or training history in those with AN is sufficient to induce athletic bradycardia; there are often early-life indications of increased physical activity and 
the involvement in high-level or competitive sport previous to the diagnosis of AN - one retrospective estimate shows a dramatic increase in activity above baseline from the age of 8 to 13 [14]. Moreover, greater childhood activity directly predicts the degree of compulsive exercise in those with AN [15]. However, committed exercise will reduce heart rate even in the short term; al-Ani \& colleagues [16] demonstrated that a drop in $12 \mathrm{bpm}$ (i.e. $69 \mathrm{bpm}$ to 57bpm) was provoked by 6 weeks of 25 min. daily exercise at $85 \%$ MHR. As above, training loads in AN are often in excess of this.

4. Significant crossover exists between non-diagnostic categories of 'exercise dependence' and 'anorexia athletica'. Both of these strongly resemble the established rodent model of AN (SIH; semi-starvation induced hyperactivity, [17], which revolves around the mutual derangement of exercise and restrictive eating behaviors. However, neither condition is clinically recognised, and can be seen as attempts to understand compulsive or excessive exercise as a secondary or related feature of AN - Bamber et al., [18] found around half a sample of chronic exercisers $(\mathrm{n}=53$ ) met criteria for disordered eating, and all who met 'exercise dependence' criteria also met formal eating disorder criteria.

\section{The current treatment approach in the management of AN is likely to resolve both}

forms of bradycardia. Inpatient treatment typically consists of 2-4 weeks of supervised refeeding, while cardiac function undergoes observation. Typically, inpatient discharge is warranted after the resolution of bradycardia [6]. However, it is important to note what cooccurs during hospitalization- a period of profound and immediate athletic detraining in which physical activity is heavily restricted or unambiguously prohibited, as it is contraindicated during periods of cardiac-related medical instability. Rodent models of detraining show straightforward increases in HR over around 2 weeks [19,20]. Likewise detraining is commonly prescribed to athletes with tachyarrhythmias or other cardiac conduction disorders related to athletic heart syndrome, resulting in partial or full reversal in both the short- and long-term $[21,22]$. In this context, one cannot presently disambiguate the relative effect of renourishment versus de-training in reversing bradycardia in AN. 
Despite this compelling overlap, the mechanisms underpinning bradycardia in AN are multifaceted. Certainly, athletic bradycardia is insufficient to explain all observed bradycardia in AN. The highest estimates of excessive exercise during AN are around $80 \%[8,15]$, thus we may presume a lower bound of at least $20 \%$ of non-exercising AN patients who may also display bradycardia, and show improvements in basal heart rate after supervised refeeding. Likewise, severe calorie restriction in the absence of exercise in animals unambiguously reduces heart rate. Most significantly, Kollai et al. [23] demonstrated a straightforward correlation between weight loss and vagal tone as measured by parasympathetic block (atropinization). The issue, rather, is how the admixture of starvation and athletic bradycardias should be understood as potential co-contributors to bradycardia in AN, since both may confer strikingly differential acute medical risk.

\section{Heart-to-Heart: Understanding Mechanisms, and Clinical Implications}

The decrease in heart rate seen in athletic bradycardia was largely assumed to be a consequence of increased cardiovagal outflow, although it is now increasingly clear that this reduction is centrally mediated by a change in intrinsic heart rate [24]. This modification is well-supported by animal models of endurance exercise showing modifications of intrinsic sinoatrial function, estimating that $40 \%$ (rats) and $76 \%$ (mice) of the training-related variance of $\mathrm{HR}$ is due to the downregulation of HCN4 [20]. In keeping, veteran endurance athletes also show altered intrinsic heart rates [25]. Cardiovagal modulation is also thought to play a role, especially as short-term changes in HR due to athletic training are reasonably reversible [26]. As such, two potential contributions are possible (exercise, starvation), leaving three potential mechanisms to bradycardia: (i) exercise increasing cardiovagal modulation, (ii) exercise decreasing intrinsic heart rate, and (iii) starvation increasing cardiovagal modulation. Separating these factors in practice, though, is not straightforward. No electrocardiographic indices have been identified which are directly relevant, and the goldstandard test remains autonomic blockade (where para/sympathetic antagonists, typically atropine and propranolol, are administered in a dose-dependent fashion). Crucially, delineating the differential 
cardiac profiles, and the differential degree of risk therein, of those with AN who demonstrate bradycardia will be a crucial endeavor as we move towards precision treatments.

Hospital admission criteria currently support an immediate admission for those with AN who demonstrate a heart rate less than 50 beats per minute, without regard to athletic activities. However, this may have unintended clinical consequences. Firstly, many endurance athletes may have resting heart rates below 50 beats per minute, and do not require medical intervention. Not accounting for athletic activity in those with AN, given the large degree of overlap, may lead to unnecessary hospitalizations. The cost of inpatient treatment in AN is high; one recent estimate [27] records the average patient stay as 32.7 days, with a daily cost of $\$ 2295$, and hospitalizations for EDs are the most costly and lengthiest of all child and adolescent mental health diagnoses [28]. Greater insight into the nature of bradycardia, especially if it demonstrates less acute medical risk and avoidable hospitalizations, would confer significant savings on health care expenditures.

Secondly, because heart rate determination is often based on a one-time snapshot at the beginning of the clinic visit [13], this measurement does not capture the variability of heart rate throughout the day, which observes a well-characterised circadian pattern in young adults [29] and can be subject to transient influences. For instance, patients with AN may acutely exercise immediately prior to vital signs measurement in clinic in order to increase their heart rate [30]. Objective and more comprehensive assessment of heart rate over longer time periods may capture more variation and better assess the risk of clinically significant bradycardia. A noteworthy point here is that those with AN typically have reduced cardiorespiratory fitness and cardiac health (lower aerobic fitness, lower maximum heart rate, reduced work capacity, etc.), although there is no reason that maximal or intense exercise even at a reduced level cannot induce or maintain athletic bradycardia.

\section{Conclusion}

A precise delineation of the factors underpinning bradycardia in those with $\mathrm{AN}$, and the relative degree of risk therein, is a crucial future endeavor in the clinical care of acute AN. To this end, determining the unique contribution of athleticism to bradycardia in the setting of AN is 
particularly important given the lack of standardized guidelines for the management of exercise in AN. Exercise is critically understudied compared to nutritional and psychopathological aspects of AN, and there is controversy in the literature even with regards to terminology ("excess exercise," "compulsive exercise," or "obligatory exercise") and measurement of exercise, which may hinder research efforts on this important topic. Clearly, additional electrophysiological and cardiological research into the development, maintenance and reversal of athletic bradycardia may inform future guidelines for the medical management of AN and individualize care based on exercise history. 


\section{Financial Disclosures}

Dr. Murray is supported by the National Institute of Mental Health (K23 MH115184) and receives royalties from Oxford University Press and Routledge. Dr. Nagata is supported by the Pediatric Scientist Development Program, funded by the American Academy of Pediatrics and the American Pediatric Society, and the Norman Schlossberger Research Fund from the University of California, San Francisco. 


\section{References}

1. Olivares JL, Vázquez M, Fleta J, Moreno LA, Pérez-González JM, Bueno M. (2005). Cardiac findings in adolescents with anorexia nervosa at diagnosis and after weight restoration. Eur J Pediatr, 164, 383-6.

2. Vázquez M, Olivares JL, Fleta J, Lacambra I, González M. (2003).Cardiac disorders in young women with anorexia nervosa. Rev Esp Cardiol, 56, 669-73.

3. Golden NH, Katzman DK, Sawyer SM, Ornstein RM, Rome ES, Garber AK, ..., Kreipe RE. (2015). Update on the medical management of eating disorders in adolescents. J Adolesc Health, 56, 370-5.

4. Yahalom M, Spitz M, Sandler L, Heno N, Roguin N, Turgeman Y. (2013). The significance of bradycardia in anorexia nervosa. Int J Angiol, 22, 83-94.

5. Sachs K, Andersen D, Sommer J, Winkelman A, Mehler PS. (2015). Avoiding medical complications during the refeeding of patients with anorexia nervosa. Eat Disord, 23, 411-21.

6. Madden S, Miskovic-Wheatley J, Wallis A, Kohn M, Lock J, Le Grange D, Jo B, Clarke S, Rhodes P, Hay P, Touyz S. (2015). A randomized controlled trial of inpatient treatment for anorexia nervosa in medically unstable adolescents. Psychol Med, 45, 415-27.

7. Drezner, J. A., Sharma, S., Baggish, A., Papadakis, M., Wilson, M. G., Prutkin, J. M., ... Corrado, D. (2017). International criteria for electrocardiographic interpretation in athletes: Consensus statement. Br J Sports Med, 51, 704-731.

8. Dalle Grave R, Calugi S, Marchesini G. (2008). Compulsive exercise to control shape or weight in eating disorders: prevalence, associated features, and treatment outcome. Compr Psychiatry, 49, 346-52. 
9. Meyer, C., Taranis, L., \& Touyz, S. (2008). Excessive exercise in the eating disorders: A need for less activity from patients and more from researchers.... European Eating Disorders Review, 16, 81-83.

10. Taranis, L., Touyz, S., \& Meyer, C. (2011). Disordered eating and exercise: Development and preliminary validation of the compulsive exercise test (CET). European Eating Disorders Review, 19, 256-268.

11. Hay, P., Touyz, S., Arcelus, J., Pike, K., Attia, E., Crosby, R., ... Meyer, C. (In Press). A randomized controlled trial of the compulsive exercise activity therapy (LEAP): A new approach to compulsive exercise in anorexia nervosa. International Journal of Eating Disorders,

12. Fagard, R. (2003). Athlete's heart. Heart, 89, 1455-1461.

13. Nagata JM, Carlson JL, Kao JM, Golden NH, Murray SB, Peebles R. (2017). Characterization and correlates of exercise among adolescents with anorexia nervosa and bulimia nervosa. Int J Eat Disord, 50, 1394-1403.

14. Davis C, Kennedy SH, Ravelski E, Dionne M. (1994). The role of physical activity in the development and maintenance of eating disorders. Psychol Med, 24, 957-67.

15. Davis C, Katzman DK, Kirsh C. (1999). Compulsive physical activity in adolescents with anorexia nervosa: a psychobehavioral spiral of pathology. J Nerv Ment Dis, 187, $336-42$.

16. Al-Ani, M., Munir, S. M., White, M., Townend, J., \& Coote, J. H. (1996). Changes in R-R variability before and after endurance training measured by power spectral analysis and by the effect of isometric muscle contraction. European Journal of Applied Physiology and Occupational Physiology, 74, 397-403.

17. Routtenberg, A., \& Kuznesof, A. W. (1967). Self-starvation of rats living in activity wheels on a restricted feeding schedule. J Comp Physiol Psychol, 64, 414-421. 
18. Bamber, D., Cockerill, I., Rodgers, S., \& Carroll, D. (2003). Diagnostic criteria for exercise dependence in women. Br J Sports Med, 37, 393-400.

19. Evangelista, F. S., Martuchi, S. E., Negrao, C. E., \& Brum, P. C. (2005). Loss of resting bradycardia with detraining is associated with intrinsic heart rate changes. Braz J Med Biol Res, 38(7), 1141-1146.

20. D’Souza, A., Bucchi, A., Johnsen, A. B., Logantha, S. J. R. J., Monfredi, O., Yanni, J., . . Boyett, M. R. (2014). Exercise training reduces resting heart rate via downregulation of the funny channel HCN4. Nature Communications, 5, 3775.

21. Biffi, A., Maron, B. J., Verdile, L., Fernando, F., Spataro, A., Marcello, G., . . Pelliccia, A. (2004). Impact of physical deconditioning on ventricular tachyarrhythmias in trained athletes. J Am Coll Cardiol, 44, 1053-1058.

22. Biffi, A., Maron, B. J., Culasso, F., Verdile, L., Fernando, F., Di Giacinto, B., . . . Pelliccia, A. (2011). Patterns of ventricular tachyarrhythmias associated with training, deconditioning and retraining in elite athletes without cardiovascular abnormalities. Am J Cardiol, 107(5), 697-703.

23. Kollai, M., Bonyhay, I., Jokkel, G., \& Szonyi, L. (1994). Cardiac vagal hyperactivity in adolescent anorexia nervosa. Eur Heart J, 15, 1113-1118.

24. Boyett, M. R., D'Souza, A., Zhang, H., Morris, G. M., Dobrzynski, H., \& Monfredi, O. (2013). Viewpoint: is the resting bradycardia in athletes the result of remodeling of the sinoatrial node rather than high vagal tone? J Appl Physiol (1985), 114(9), 13511355.

25. Stein, R., Medeiros, C. M., Rosito, G. A., Zimerman, L. I., \& Ribeiro, J. P. (2002). Intrinsic sinus and atrioventricular node electrophysiologic adaptations in endurance athletes. J Am Coll Cardiol, 39, 1033-1038. 
26. Iwasaki, K., Zhang, R., Zuckerman, J. H., \& Levine, B. D. (2003). Dose-response relationship of the cardiovascular adaptation to endurance training in healthy adults: how much training for what benefit? J Appl Physiol, 95, 1575-1583.

27. Guarda AS, Schreyer CC, Fischer LK, Hansen JL, Coughlin JW, Kaminsky MJ, ... Redgrave GW. (2017). Intensive treatment for adults with anorexia nervosa: The cost of weight restoration. Int J Eat Disord, 50, 302-306.

28. Bardach NS, Coker TR, Zima BT, Murphy JM, Knapp P, Richardson LP, Edwall G, Mangione-Smith R. (2014). Common and costly hospitalizations for pediatric mental health disorders. Pediatrics, 133, 602-9.

29. Bonnemeier H, Richardt G, Potratz J, Wiegand UK, Brandes A, Kluge N, \& Katus HA. (2003) J Cardiovasc Electrophysiol,14, 791-9.

30. White DW, Raven PB. (2014). Autonomic neural control of heart rate during dynamic exercise: revisited. J Physiol, 592, 2491-500. 